\title{
Tratamiento odontopediátrico integral en parálisis cerebral. Reporte de un caso.
}

Odontopediatrics integral treatment in cerebral paralysis.

\author{
Resumen \\ Se describe el casu de una niña de 4 años 3 meses de edad, con parálisis cerebral \\ discinética con tendencia espástica causada por citomegalovirus con síndrome \\ convulsivo, quien fue referida a la Clínica Integral del Niño de la Facultad de \\ Odontología de la Universidad Nacional Mayor de San Marcos (UNMSM) para \\ su tratamiento. \\ La paciente fue evaluada de manera integral con procedimientos odontológicos \\ no convencionales sin sedación consciente, teniendo cn cuenta la edad y el nivel \\ de colaboración que presentaba, además de trabajar con un equipo \\ multidisciplinario médico-odontológico. \\ Se destaca el echo que la paciente redujo significativamente la frecuencia de \\ convulsiones luego del tralamiento odontológico, finalmente se resalta la \\ importancia de integrar al odontólogo en el equipo multidisciplinario que trabaja \\ con estos pacientes.
}

\begin{abstract}
Report a case of girl of 4 years 3 months with congenital cerebral palsy distonic caused by citomegalovirus infection with convulsive sindrome. The patient was referred to the pediatric dentistry departament at the dental school of the Universidad Nacional Mayor de San Marcos (UNMSM) for her odontopediatric treatment.

The patient had an Integral evaluation and treatment with no conventional dental procedures, without consciencious sedation. Haviry in consideration her age and cooperation level. Several medical and dental specialities was included. A significative diminishment in the convulsive frecuency after the dental treatment was referreal.

Finally the of importance integrate odontology into multisciplinary team that works with this kind of patient is remarked.
\end{abstract}

\section{Introducción}

Parálisis cerebral, es un término usado para describir un grupo de trastornos crónicos que aparecen durante los primeros años de vida ${ }^{1}$, relacionados con cl control del movimiento y la postura, debido al daño de una o más partes del cerebro que controlan e] movimiento $0^{2}$ y que por lo general, no empeora con el tiempo ${ }^{1,2}$. El término "cerebral" se refiere a los hemisferios cerebrales afectados y "parálisis» describe cualquier trastorno que limite el contrul del movimiento 1 .

Las personas con parálisis cerebral presentan problemas como retraso mental, convulsiones, epilepsia, problemas de crecimiento, aprendizaje, visión, audición y habla ${ }^{1,2}$, sensibilidad y percepción anormales ${ }^{1,2}$, reflujo gastroesofágico y problemas respiratorios ${ }^{3,4}$.

Existen cuatro categorías amplias de parálisis cerebral según el tipo de per- turbación en el movimiento: espástica, discinética, atáxica y formas combinadas ${ }^{1,-3}$.

La parálisis cerebral no es una enfermedad con etiología única, es más bien un grupo de trastornos relacionados entre sí que tienen causas distintas ${ }^{1.3}$ que ocurren durante el embarazo o cerca del momento del nacimiento y que pueden interrumpir el desarrollo normal del cerebro? En el $70 \%$ de los casos el daño cerebral tiene lugar antes del nacimiento, en la minoría, el daño se da cerca del parto, durante los primeros meses o años de vida y en muchos otros se desconoce la causa ${ }^{2}$. Entre las principales causas tenemos: Infecciones durante el embarazo (Algunas infecciones contraídas por la madre, como rubéola, citomegalovirus y toxoplasmosis, pueden producir parälisis cerebral $\left.{ }^{1,2}\right)$, asfixia perinatal, parto prematuro, complicaciones durante el parto y nacimiento, intolerancia de
Artículo Clínicos

\section{Gabriel Nima Bermejo ${ }^{1 *}$; Mariela Ruthsana Romero Velarde ${ }^{2^{*}}$}

\author{
Bachiller en Odontología \\ Departamento Académico de Estomatología \\ Fediátrica \\ - Facultad de Odontología, Universidad \\ Nacional Mayor de San Marcos, Lima, Perú \\ E-mail: gabrieln@mixmail.con
}

Palabras clave: parálisis cerebral, Citomegalovirus, Tratamiento odontopediátrico integral, Manejo discapacitados, Síndrome convulsivo, Trabajo multidisciplinario.

Key words: cerebral palsy, Citomegalovirus, Integral pediatric dental treatment, Disabilities treatment, Convulsive sindrome, Multidisciplinary team.

Rh, ictericia en los infantes, apoplejía o hemorragia intracraneal y parálisis cerebral adquirida ${ }^{1,2}$.

El diagnóstico se realiza principalmente evaluardo la manera como se mueve un bebé o niño pequeño, evaluando el tono muscular, preferencia temprana por una mano y la persistencia de ciertos reflejos primitivos ${ }^{1,2}$. Son útiles las imágenes de resonancia magnética y tomografías computarizadas ${ }^{1,2}$.

El tratamiento médico de la parálisis cerebral está a cargo de un grupo de profesionales 1, 2, compuesto por un médico, ortopeda, terapeuta físico, terapeuta ocupacional, foníatra, asistenta social, psicólogo, educador, y oftalmólogo; los cuales deben iniciar un tratamiento temprano y continuo ${ }^{1,2}$.

No existen anomalías intraorales exclusivas de personas con parálisis cerebral. Sin embargo varias patologías orales son más frecuentes o más graves que en la población normal 5 , 
como la presencia de enfermedad periodontal y el alto número de caries pudiendo deberse a la mala higiene y al régimen alimenticio (dieta blanda y rica en carbohidratos) ${ }^{3 \cdot 7}$. Otros problemas descritos en son: hipoplasia del esmalte, mordida abierta, maloclusiones, bruxisno, respiración bucal y protrusión del tercio medio bucal ${ }^{3-\gamma}$.

\section{Caso clínico}

Paciente de sexo Femenino de 4 años 3 meses de cdad, procedente de Lima, derivada del Instituto Nacional de Rehabilitación, a la Clínica de Odontopediatría de la Facultad de Odontología de la UNMSM para su tratamiento odontopediátrico integral (Fig. 1). Presenta parálisis cerebral discinética con tendencia espástica causada por citomegalovirus.

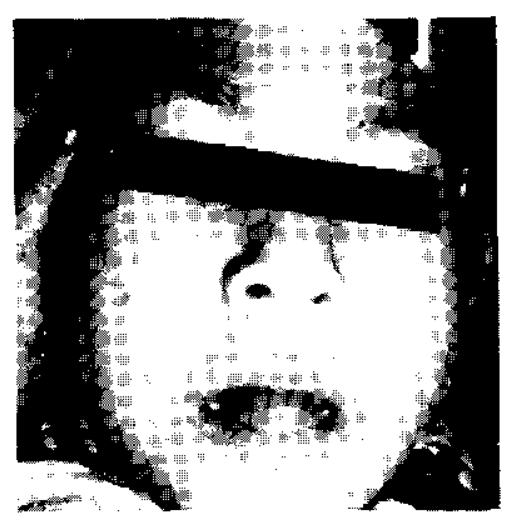

Fig. 1: Paciente de sexo femenino de 4 años 3 meses de edad, con diágnostico de Parálisis Corebral de discinética con tendencia espástica

A] confeccionar la historia clínica la madre afirma que durante el sexto mes del embarazo presentó adenopatía. Su enfermedad fue diagnosticada a los ocho meses de nacida al notar que la niña no podía sentarse; padece de convulsiones, su primer episodio convulsivo ocurrió cuando tenía un año ocho meses de edad, los que aumentaron gradualmente hasta llegar a $60 \mathrm{cn}$ dos horas a la edad de 2 años 8 meses, la enfermedad se encuentra controlada, las convulsiones se presentan con una frecuencia de un mes $\sin$ causa predisponente alguna, con menor intensidad; hospitalizada hace aproximadamente año y medio por presentar síndrome hemolítico urémico, en dicha circunstancia se realizó diálisis y transfusión de sangre, no existen problemas de visión y audición.
Con una dieta compuesta básicamente por papillas y alimentos licuados. Paciente con tratamiento odontológico previo, a la edad de 3 años 8 meses.

Su medicación actual está com-puesta por Ácido Valproico $450 \mathrm{mg}$. (Valpakine(i)) en tres tomas diarias, Fenobarbital $100 \mathrm{mg}$. en dos tomas y Clonazepan $1.8 \mathrm{mg}$. (Rivotril(B) en tres tomas, hasta hace un año tomaba Fenitoina (Epamin( $($ )).

Ectoscópicamente se observó imposibilidad de caminar, hablar, sostener la cabeza, sialorrea, cara simétrica y larga, frente amplia, cejas pobladas, lengua afuera y boca abicrta (Fig. 1).

Intraoralmente se observó encías y mucosa rosado coral sin alteraciones, paladar profundo, arcada en forma de «U», arrugas palatinas prominentes, lengua con lesiones eritematosas de bordes blanquecinos de ubicación variada, saliva de secreción abundante de consistencia viscosa, reflejo de morder exagerado y deglución atípica.

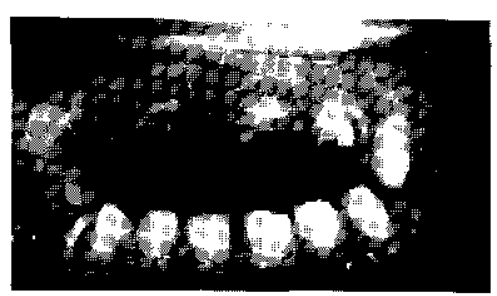

Fig. 2: Vista intraoral anterior en oclusión antes del tratamiento. El color rojizo de la encia sie deber a defectos de la fotengrafía.

Al examen dental se observó dentición temporal completa, múltiples lesiones cariosas en el sector anterosuperior (de color negro y consistencia blanda llegando a destruir casi por completo la corona clínica (Fig. 2 y 3) y en todas las piezas del sector posterior; mordida abierta anterior y una imposibilidad para realizar movimientos de lateralidad de la lengua y mandíbula.

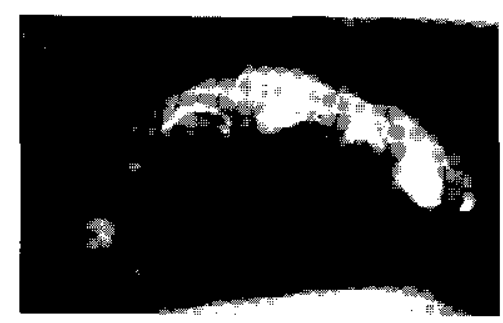

Fig. 3: Foto uclusal superior antes del tratamiento. El color rojizo de la encía se debe a defectos de la fotografia
Se tomaron radiografías oclusales superior e inferior y periapicales de los cuatro cuadrantes, sin encontrarse alteración en los gérmenes dentarios, se encontró reabsorción radicular externa atípica en el tercio apical de los incisivos centrales decíduos (Fig. 4).

Luego de una evaluación detallada se llegó al siguiente diagnóstico: Paciente con riesgo estomatológico alto (por imposibilidad para realizarse una buena higiene y dieta adecuada), lesiones de caries dental: Leves en 53 (V), 63(V); moderadas en 55 (O), 54 (O), $64(0), 65(0), 75(0), 84(0), 85(0)$, y destrucción de la corona clínica en $52,51,61,62$; mordida abierta anlerior relacionado a deglución atípica, se descartaron problemas de origen respiratorio.

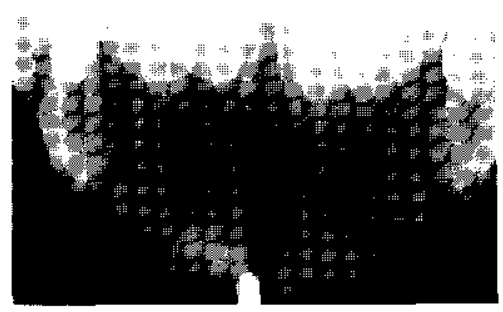

Hig. 4: Oclusal superior. Observese la reabsorción radicular de los incisivos centrales, asi como la radinlucide del hueso alveulat.

Para brindar un tratamiento integral se planteó los siguientes objetivos: motivación e instrucción a los padres para el correcto cepillado de los dientes, terapia de flúor en dosis de ataque con flúor silano al 1\% (Flúor Protector $(\mathbb{k})$ en topicariones semanales durante un mes, restauraciones con resina fluida de las lesiones de caries leves, rehabilitación con coronas de resina en los incisivos laterales superiores, exodoncia de los incisivos centrales y las piezas restantes serían restauradas con ionómero de vidrio. Se realizó una pulpotomía en la pieza 64, por exposición pulpar durante el Iratamiento cambiando el ionómcro que se tenía pensado colocar por una amalgama adhesiva ${ }^{8}$; el material de restauración final usado para las piezas restantes fue Vitremer(B) colocándose Ketac Molarß sólo en una pieza (74).

\section{Discusión}

Cerca del 10 al $20 \%$ de las personas con parálisis cerebral padece la forma discinética, esta forma se caracteriza por fluctuaciones en el tono muscular (que pasa de demasiado duro a demasiado blando), y que 
aveces se asocia con movimientos descontro-lados ${ }^{32}$. En la paciente el tono muscular era espástico la mam yor parte del tiempo, estos pacientes son llamados atetósicos «tensos» $y$ suelen ser confundidos con los espásticos pero se diferencian de ellos fundamentalmente por la presencia de movimientos involuntarios de tipo isotónico'.

La infección de citomegalovirus (CMV) generalmente no produce sintomas en los niños y adultos infectados ${ }^{10}$. La mujer puede transmitir el CMV a su bebé antes del nacimiento, durante el parto o al amamantarlo, pero es poco común que sufran problemas graves relacionados con el vírus ${ }^{10}$, (cerca del $90 \%$ de los bebés infectados no presenta sintomas al nam $\left.\operatorname{cer}^{10}\right)$, sin embargo, hasta un $15 \%$ de ellos desarrolla una o más anomalias neurológicas, como retraso mental, problemas de aprendizaje, pérdida auditiva o de la vista y otros parálisis cerebral'. Las mujeres que contraen la infección de CMV por primera vez durante el embarazo tienen un riesgo del 30 al $40 \%$ de transmitirlo al feto y si son infectadas por primera vez en el tercer trimestre tienen más proba bilidades de transmitix la infección a su bebé que las mujeres infectadas en una etapa anterior del embarazo ${ }^{10}$.

Las personas con Parâlisis cerebral no presentan alteraciones intraorales patognomónicas ${ }^{3,5}$, las patologías bucales más comunes en ellos son las mismas que afligen a la población general, algunas de ellas más exacerbadas debido a las dificultades motoras que presentan ${ }^{3,5}$.

Los niños con parálisis cerebral son propensos a una mala salud bucal debido a los problemas psicomotores, dieta cariogénica y difícil atención que presentan ${ }^{\mathrm{k}}$.

La enfermedad periodontal afecta con mayor frecuencia a estos pacientes debido a la falta de una buena higie$\mathrm{ne}^{5,3}$, pues son incapaces de cepillarm se los dientes y esto agregado a los medicamentos que toman ${ }^{35}$.

La elevada cantidad de lesiones cariosas es causada por la necesaria dieta altamente cariogénica y las dificultades de masticación e higiene . $^{3.5 .5}$. Los problemas que causan una dificil alimentación, son la protrusión lingual, el reflejo de morder exagerado, el reflejo de vómito anormal e hipersensibilidad en el área alrededor de la boca ${ }^{3}$.
La prevalencia de malociusiones es aproximadamente el doble que en la población en general s. Entre los trastornos que se observan con mayor frecuencia encontramos la protrusión de los dientes anterosuperiores ( por la posición protruída de la lengua sobre los procesos alveolares de los incisivos superiores ${ }^{12}$, paladar pequeño, over bite y over jet alterados, mordida abierta y cruzada unilate$\mathrm{ra}^{5,12-14}$, tendencia hacia la relación molar clase II $^{12-14}$; existiendo una directa relación entre las actividades musculares anormales de la cabeza y cuello, el bruxismo, las fuerzas intraorales, la posición de la lengua, respiración bucal y la deglución atípica ${ }^{12-15}$. La prevalencia de maloclusiones pueden explicarse por la adaptación postural craneocer. vical, que responde a la necesidad de mantener el suficiente espacio nasofaringeo para el desarrollo de las funciones básicas estomatológicas como la respiración, deglución y fonoarticulación ${ }^{13}$; la morfología craneofacial puede explicar las características motoras masticatorias que parecen estar asociadas con alteraciones en el funcionamiento de la articulación temporo-mandibular y con una disminución en la presión de mordida ${ }^{12,}$ 14,15 .

Algunos pacientes presentan un bruxismo severo que ocasiona una pérdida de dimensión vertical oclusal y problemas de $\mathrm{ATM}^{3,5}$. También se ha encontrado una alta incidencia de hipoplasia del esmalte ${ }^{3,5,5}$, retraso en la erupción de dientes permanentes, que los hace más susceptibles a desarrollar maloclusiones $\mathrm{s}^{6,11}$.

Estas personas son más susceptibles a traumatismos sobre todo en los dientes anteriores debido a la acentuada protrusión de los maxilares ${ }^{3,5,6}$ y a las dificultades para caminar que ocasionan caídas constantes ${ }^{3,5,6}$.

El tratamiento odontológico implica muchas veces el tratamiento de pacientes convulsivos, se habla de una incidencia de $50 \% \%^{3}$, por esto es necesario establecer un vínculo entre el neuropedriatra para obtener información detallada con respecto a la enfermedad, medicación y tipo de medicamentos que pueden ser usados en el paciente

El uso de anticonvulsivantes sobre todo del grupo de la fenitoína puede causar algunas alteraciones estomatológicas agregadas en pacientes que padecen de convulsiones, la literatu- ra especializada sólo refiere ef́ectos tóxicos de hiperplasia gingival como única manifestación bucal $3,5,16,07$, Sin embargo, Meráz (1998) ${ }^{17}$, encontró otras alteraciones como por ejemplo glositis migratoria benigna $(30.8 \%$, paladar profundo $(30.8 \%)$, arrugas palatinas prominentes $(46.2 \%)$, desmineralización e hipoplasia del esmalte $(69.2 \%)$, caries $(30.8 \%)$, maloclusión $(30.8 \%)$, reabsorciones oseas y radiculares atípicas, imitación de la apertura bucal $(46.2 \%)$, labios resecos $(53.8 \%)$, agrandamiento del conducto de Stensen $(53.8 \%)$, además de hiperplasia gingival (30.8\%) entre otras en pacientes que consumian estos fármacos ${ }^{17}$. El valproato de sodio puede ocasionar también. hiperplasia gingival ${ }^{3}$.

Al examen estomatólogico intraoral se observó en la paciente glositis migratoria benigna, paladar profundo, rugas palatinas prominentes, desmineralización e hipoplasia del esmalte, caries, maloclusión, reabsorción ósea y radicular atipica, el uso de medicamentos anticonvulsivantes puede explicar en parte algunas alteraciones encontradas ${ }^{77}$. La incontinencia salival, estaría más relacionada con la imposibilidad de deglutir y pasar la saliva de la boca antes que con una secreclón exagerada de las glândulas salivales ${ }^{3}$, una caracteristica especial de esta saliva fue su viscosidad, dificultando la aplicación del fluor barniz debido a que se adhería a los dientes. La imposibilidad de realizar movimientos de lateralidad tanto de la lengua como de la mandibula parece estar relacionado con disfunción de la $\operatorname{ATM}^{13,15}$,

Las dificultades de alimentación en estos pacientes se deben fundamenm talmente a las dificultades de masticación que presentan, en la paciente se encontró una deglución atípica con reflejo de morder exagerado e imposibilidad para masticar. En general las personas que padecen este tipo de parálisis cerebral tienen una alta probabilidad a desarrollar una disfagia del segundo tiempo; el reflejo inh:bitorio masetérico (RIM) (reflejo responsable de regular las cargas masticatorias ante diferentes materiales comestibles, así como proteger a las estructuras bucales de fuerzas inesperadas ${ }^{18}$ ) muchas veces se halla alterado en estos pacientes y sus características son explicadas por las condiciones estomatologícas, fundamentalmente por el número de contactos dentarios 19. Estos hallazgos 
son un indicio para priorizar la prevención, a las alteraciones clínicas cstomatológicas que provoquen disminución de Ios contactos denta$\operatorname{rios}^{15,19}$.

La evaluación radiográfica se realizó ayudados por una pinza hemostática para contrarrestar los movimicntos involuntarios (Fig. 5). Obtener una radiografía panorámica ${ }^{3}$, hubiera sido lo ideal pero no fue posible debido a la poca colaboración de la pariente que obligaba a usar sedación consciente o anestesia general para

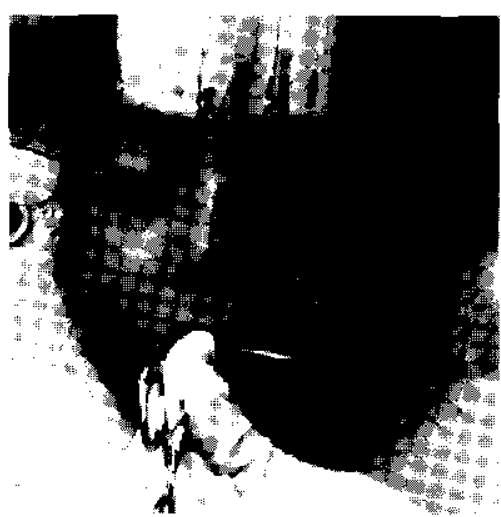

Fig. 5: Evaluación radiográfica. Se utilizo una pinza hemostática para contarrestar los movimientos de la paciente?

lograr tal fin, por lo que se prescindió del procedimiento.

Debido a la imposibilidad de la niña de realizarse un correcto cepillado se instruyó a sus padres utilizando una técnica horizontal ${ }^{11}$ (esta técnica es una modificación de la de Bass, las cerdas del cepillo se colocan a $90^{\circ}$ con respecto a los dientes ${ }^{6}$ ), empleándose un cepillo de cabeza pequeña ante la falta de un cepillo eléctrico ${ }^{2,5,11}$.

Para el mancjo del paciente se utilizó restricción física mediante un Macri (Fig.6), el Papoose Buard era el medio de restricción ideal pero ante la falta de éste, el Macri funcionó muy bien; se evitó el uso de ancstesia general o sedación consciente por las convulsiones del paciente, además de ello en ningún procedimiento se usó aislamiento absoluto, pero sí un abrebocas de Mc Keeson en los casos estrictamente necesarios y con el cuidado respectivo; aunque algunos autores recomiendan el uso de aislamiento absoluto mientras que evitan el uso de un abrebocas para prevenir problemas de deglución ${ }^{5,20}$. En los pacientes que presentan convulsiones tanto el aislamiento absoluto, abre bocas, portamatriz y otros instru- mentos deben ser usados sólo en casos extremadamente necesarios puesto que puede existir la necesidad de ser removidos inmediatamente si se presenta una crisis convulsiva ${ }^{3}$. Para una mejor exploración se presionó ligeramente con el indice el borde an-

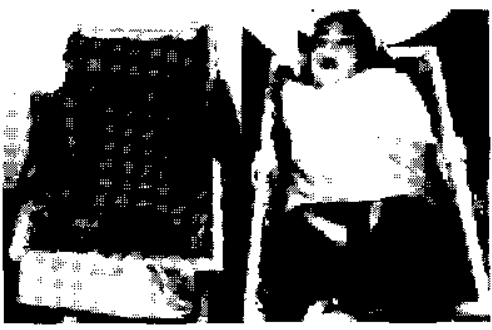

Fig. 6: Para la atención de la paciente so utilizo un Macri (A), que nos permitio controlar los movimientos involuntarios de la niña (B) y lograr una atención más räpida.

tcrior de la rama ascendente $y$ en el trígono retromolar, esto facilitó la apertura bucal ${ }^{2 c}$.

El riesgo estomatológico alto obligó usar un esquema de fluorización en dosis de ataque con Flúor silano al $1 \%$ en barniz con aplicaciones semanales por un mes $y$ luego cada tres meses, esta elección para evitar problemas de deglución al utilizar otro tipo de flúor, el barniz de flúor brinda resultados óptimos en estos pacientes $^{3}$

Existía un gran contraste entre los incisivos superiores en los que-la caries habia tomado toda la corona cl $j-$ nica frente a los inferiores que no presentaban ninguna lesión cariosa (Fig. 2 y 3), esto pudo deberse a que la gran mayoría de estos pacientes padecen de Reflujo Gastroesofágico $(86 \%)^{4}$; Según la madre la niña padeció hasta los 2 años de constantes regurgitaciones y vómitos pudiendo ocasionar estas caries, el hábito de biberón nocturno es muy común en estos pacientes $^{3}$.

Se realizó las extracciones de los incisivos centrales superiores por la reabsorción que tenia el hueso alveolar además la reabsorción radicular externa atípica de estos dientes los hacía más propensus a una fractura apical durante un evento convulsivo (fig. 4 y 7 ), poco antes de las exodoncias el número de convulsiones aumentó notoriamente, la madre había notado que durante ellas la niña llevaba las manos a esta zona, luego de las extracciones han desaparecido, hasta la elaboración de este artículo no se han vuelto a presentar hace ya

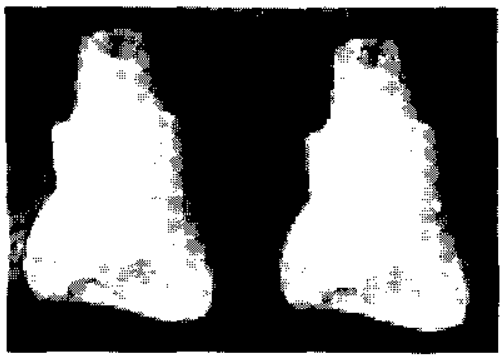

Fig. 7: Piezas exlraidas, se observa claramente reabsorción del tercio apical. Fotografía tomada una semana después de lo exodorcia.

cinco meses.

Las piezas 52 y 62 se rehabilitaron con coronas de resina (mediante técnica propia del autor, que consiste en la fabricación de cofias de resina que luego serán cementadas); no se fue necesario el tratamiento pulpar para lograr una mejor retención debido a que las coronas de los dientes temporales son anchas en su porción más gingival y que la longitud cérvico incisal es corta, la restauración tendrá una consistencia maciza, fuerte, que la hará resistente a las fracturas ${ }^{21}$; este procedimiento nos dio las ventajas de mejorar la fonación, deglución, el acto masticatorio, lograr una mejor oclusión, conservar las raices de los dientes lemporales, los permanentes conservan su guía de erupción normal, evita el uso de mantenedores de espacio y se recupera la estética (Fig. 8) ${ }^{21}$.

Durante el tratamiento de operatoria

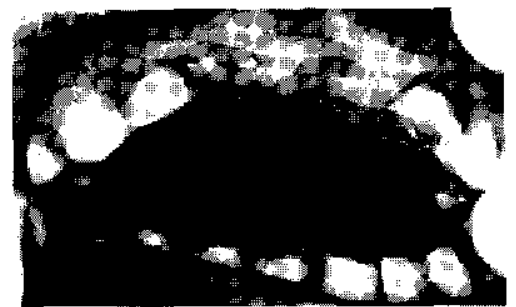

Fig. 8: Vista intraoral unmediatamente después de la colocación de coronas de resina en piezas 52 y 62, con buenus resultados estéticos.

dental se encontró un esmalte y dentina muy lábiles desgastados fácilmente por las tresas, debido a una hipocalcificación que posiblemente este influenciada por la medicación administrada, se sabe que la fenitoína altera el metabolismo de la vitamina $D^{16,17}$

Se optó por el ionómero de vidrio como material de obturación definitiva en el sector posterior por su liberación constante de flúor, fácil técni- 
ca de colocación frente a otros materiales como la resina y amalgama, adhesion al diente y alta biocompatibilidad ${ }^{3}$, además de obtener un excelente resultado estético (Fig.11) inicialmente se utilizo ionomero para la técnica TRA (Ketac Molar(i) pero se cambió por ionómero de restauración de fotocurado (Vitremer(B) reduciendo el tiempo de trabajo.

Debido a la profundidad de una de las carics se realizó una pulpotomía (se encontró una cámara pulpar bastante amplia); ante la imposiblidad de realizar una rchabilitación convencional con una corona de acero, por la poca colaboración del paciente (que dificultaba un buen tallado y adapta ción de la misma), una altura ocluso gingival corta y el tiempo que deman daría, se optó por rehabilitar la pieza con una amalgama adhesiva ${ }^{8}$, permitiendo acortar el tiempo de trata miento y obtener un diseño de la cavidad menos exigente; debido al bruxismo se introdujeron dos modificaciones en la técnica, que consistieron en hacer un pequeño surco a 1 mm del borde cavo superficial con una fresa de rueda pequeña para favorecer la retención y colocar sólo amalgama en la última capa de la obturación logrando un tallado y brunido más rápido, además de una mayor dureza en la restauración.

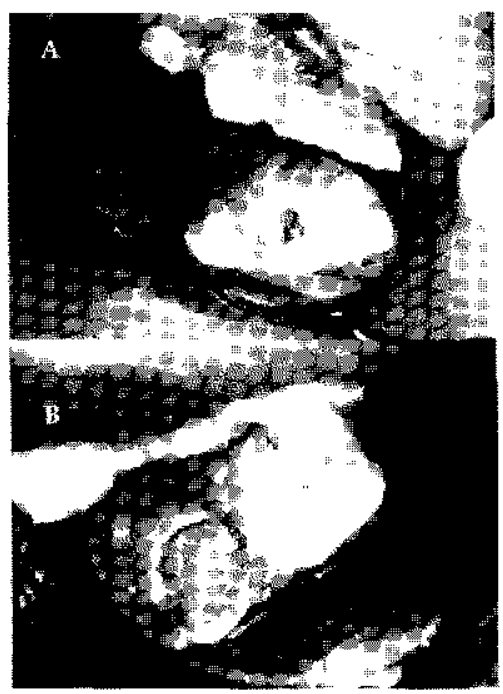

Fig. 9: Pulpotomia en la pieza 65 (A) se restairo con una amalgama adhesiva (B) Ios bordes desbordantes fueron corregidos con el pulido.

Cun respectu a la mordida abierta anterior el trakamiente indicado para este caso es el empleo de una rejilla, no se colocó porque puede ser un estimulo que origine nuevas convulsiones en la paciente. En pacientes con parálisis cerebral el tratamiento de ortodoncia con brakets está contraindicado por la dificultad de higienización, pero los aparatos removibles y fijos como expansores, mantenedores de espacio y la extracción seriada pueden ser muy útiles ubteniendo buenos resultados ${ }^{3}$.

Se realizó una inzerconsuita con el neuropediatra pata conocer algunas consideraciones especiales en el tralamiento, indicando que podiamos hacer uso de cualquier antibiótico y AINE, respecto al uso de la anestesia local nos indico aumentar una gota más de Clonazepan en la dosis del dia para evitar problemas de convulsiones. Durante el tratamiento se utilizó Clorhidrato de Mepivacaína y levonordefrína (Carbocaine $2 \%$ con NeoCobefrin 1:200000) cumo anestésico local en caso de dolor se prescribió Paracetamol (Panadol Infantil(s) el ÁcidoValproico usado por el paciente imposibilita el empleo del buprofeno ${ }^{16}$.

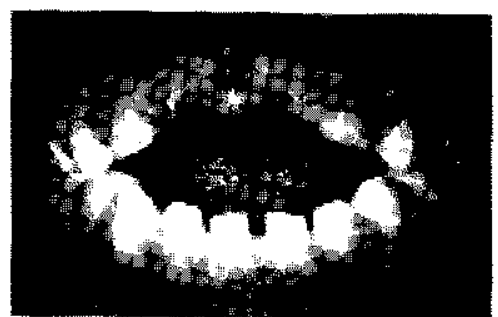

Fig. 10: Vista intraoral anterior en ulusión después del tratamiento.
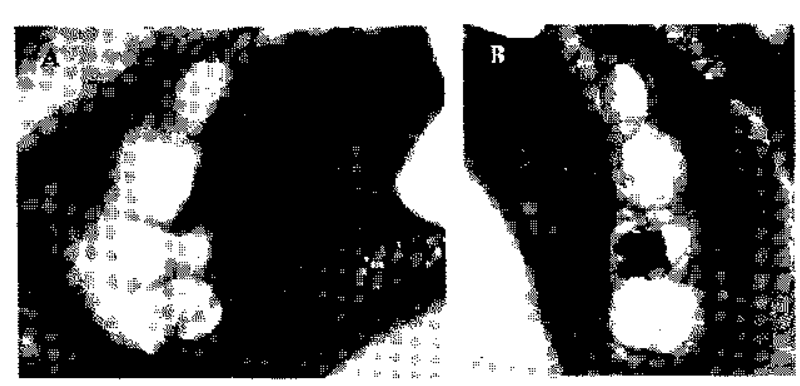

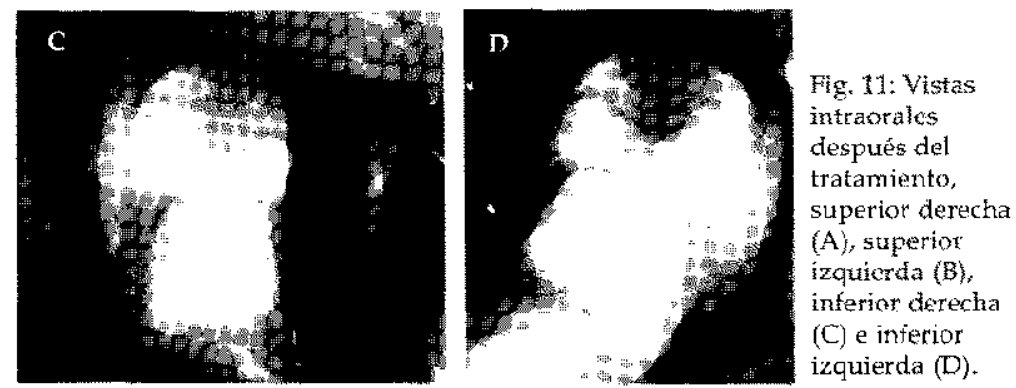

El tratamiento odontológico en un principio no es diferente en un paciente con parálisis cerebral, la gran diferencia está en la forma del manejo y abordaje del paciente que exige de parte del profesional agilidad para realizar los diferentes procedimientos sin perder las condiciones de seguridad y calidad ${ }^{3}$.

Desde el punto de vista protesional varias situaciones deben ser analizadas, nítidamente las manifestaciones clínicas que posee el paciente, como retraso mental o desajuste emocional, incapacidad de andar o sentarse, disfunciones sensoriales e incapacidad para las actividades de la vida diaria, las que dificultan el tratamiento ${ }^{3}$.

La odontología durante muchos años ha fomado una posición reticente, a veces indiferente, otras negativa $y$ hasta iatrogénica a la hora de ofrecer al paciente especial, la anestesia general como única alternativa de atención ${ }^{22}$. Casi el $80 \%$ de odontopediatras en los Estados Unidos utilizan sedación consciente para el tratamiento en estos pacientes, dejando de lado el control de la ansiedad; este medio promueve una mayor colaboráción por parte del paciente, es una técnica de mucho menur costo comparada con la sedación consciente y la anestesia general ${ }^{3}$, permitiendo lograr que más pacientes tenga acceso a un tratamiento odontológico sobre todo en países subdesarrollados como el nuestro, el facultativo no debe considerar el tiempo desperdiciado en obtener con el paciente una interacción adecuada para lograr una empatía la cual rendirá frutos preciosos en un futuro ${ }^{7}$, además mu- 
cho más allá del riesgo quirúrgico que implica la aplicación de sedación consciente $o$ anestesia general, la evidencia clínica con respecto a estos procedimientos revela que los logros obtenidos por $\mathrm{cl}$ paciente gracias al trabajo constante de equipos multidisciplinarios desaparecen o bien retroceden a etapas iniciales luego de la aplicación de estas técnicas ${ }^{22}$.

La prevención es la metodología más lógica para mejorar la salud oral en estos pacientes esto va a depender de un gran entendimiento por parle del niño lo que dependerá del tipo de parálisis cerebral que padezca y del interés que muestren los padres o tutores ${ }^{3,5}$. Los programas de odontología preventiva son más importantes para los niños discapacitados que para los niños sanos, debido a que sus faclores predisponentes hacen que la asistencia dental restauradora, sea màs difícil.

La prevención se debe dar en tres campos, control de dieta y nutrición, control de higiene y procedimientus preventivos, tanto en el consultorio, en casa y en la institución que esta frecuentando ${ }^{3}$.

Finalmente la labor del odontólogo es de suma importancia, pues el mantener una optima salud oral permitirá al paciente obtener un mejor nivel de nutrición lo que redundara finalmente en un mejor desarrollo y desempeño en sus diversas terapias y actividades.

\section{Referencias}

1.Parálisis Cerebral. Obtenible en Instituto Nacional de trastornos Neurológicos y Apoplejía» <www.ninds.nih.gov> [consulta: 12 Julio 2003]

2.Parálisis Cerebral. Obtenible en March of Dimes Resource Center. <www.racersano.org/pdf/ paralisiscerebral.pdi> [consulta: 06 Agosto 2003]

3.Costa S., Angela M.; Ferraretto, Ivan. Paralisia Cerebral: Aspectos práticos. Asociación Brasilcira de parálisis Cerebral. Sao Paulo: Memnon, 1998.

4.Barrón, Fabiola; Riquelme, Mano; Elizondo, Jorge; Quimbar, Alberto. Reflujo Gastroesofágico y Problemas
Respiratorios en Parálisis Cerebral In fanlil. Experiencia del Instituto Nuevo Amanecer. Obtenible en: Instituto nuevo amanecer <htp:// www.nucvoamanecer.edu.mx/ medica/invest_publicaciones.asp>

5.Weddell, James $\Lambda . ;$ Mc Kown, Carol; Vash B., Jones J.: Problemas Odontológicos de Ios niños Discapacitados. Fn: Mc. Donald, R y Avery D.: Odontología Pediátrica y del Adolescente, $6^{\circ}$ Ed., Madrid, Ed. Musby - Duyma Libros, 1995.

6.Morales H., Carmen C. Parálisis Cerebral: sus efectos sobre la erupción del primer molar permanente y las condiciones de salud-enfermedad. Acta odontol. venez; 1987, 25(1):13 33.

7.Pirela M., María A.; Salazar V., Carmen R.; Manzano F., Moisés A.: Patología bucal prevalente en niños excepcionales. Acta odontol. venez; 1999, 37(3):193-8.

8.Aguilar G., Nenisse: Amalgama adhesiva en Odontopediatría. Rev. Gaceta Odontología, 2000; 2(5) 8-11.

9.Segovia, María L.: Interrelaciones entre la oduntuestomatología y la fonoaudiología - La deglución atípica. $2^{\circ}$ Edición, Madrid, Ed. Médica Panamcrican, 1988.

10.Infección de Citomegalovirus durante el Embarazo. 2001,obtenible en March of Dimes Resource Center. $<$ www.nacersano.org/pdf/ ParalisisCerebral.pdf $>$ [consulta: 06 Agos to 2003]

11. Camacho A., Marianela. Fil uso de un cepillo dental modificado en niños con Parálisis Cerebral. 2002, Oblenible en Asuciación Costricence de odontolólogos: <htp:// www.dentalaccocr.com/es/revistas/ $2002 / a r t 17 /$ hoja 005 .html= [Consulta: 27 Agosto 2003]

12.Alfaro M., Patricia; Cedeño P., Enrique; Valderrama D., Jaqueline.: Condiciones Cefalomé-tricas en parientes con Parálisis Cerebral. Temas Selectos de Investigación Clínica, 1997; III (X):103-113.

13.Alfaro M., Patricia; Gonzâles G., Hortensia; Sánchez R., Jesús.; Nuño L., Alberto;Angeles M., Fernando; García R., Jaime; Galicia A. Araceli; Osomo E., María C.: Reflejo Inhibitorio Masetérico y Condiciones Clínicas Estomatológicas en Pacientes con Parálisis Cerebral; Rev. Arch Neurociencia, 1999; 4(4):175-182.
14.Alfaro M., Patricia; Gonzáles G., Hortensia, García R., Jaime.: Reflejo Inhibitorio Masetérico, su Contexto Nexurofisiologico y Avances de su evolución en la Parálisis Cerebral; Temas seleclos de Investigación Clínica, 1995; I(1):79-103.

15.Alfaro M., Patricia; Gonzáles G., Hortensia; Angeles M., Fernando; Nuño L., Alberto; Osorno E., María C; García R., Jaime; Galicia A., Araceli.: Control Voluntario de la Fuerza de Mordida en pacientes con Parálisis Cerebral y con disfunción de la Articulación Temporomandibular. Rev. Med. Oral, 1999; 1(2):53-57.

16.Brian J. Sanders, James A. Weddell, Nancy N. Dodge. Mariging Patientes who have seizure disorders: dental and medical issues. JADA, 1995, Dec 1641 - 1647

17.Meráz A., Héctor F.; Alteraciones bucales encontradas en pacientes epilépticos que consumen fármacos del grupo fenitoína (difenilhidatoina) (Dilantín, Fpamin). Estudio Transversal descriptivo con apoyo estadístico. Rev. ADM, 1998; $55(1): 29-33$

18. Osorno E., Carmen; Alfaro M., Patricia; Nuño L., Albertu; Ángeles M., Fernando; Descripción y corrclacioncs de los componentes del reflejo inhibitorio masctćrico en niños sanos. Temas Selectos de Investigación Clínica, 1999; V (X):5362.

19.Alfaro M., Patricia; Osorno F., María C.; Nuño L., Alberto; Ángeles M., Fernando.: El reflejo inhibitorio masetérico en niños y adultos sanos, parálisis cerebral y disfunción de la articulación tcmporomandibular; Rev. Arch. Neurocien, 2002; 7(3):136-141

20.Camerón, $\Lambda$, Widner, Richard. Manual de Odontología Pediátrica. Ed. Harcourt Brace, 1998.

21. Franco T., Francisco J. Reconstrucción coronaria de dientes deciduos con resina de fotocurado. 2001, obtenible en Odontología On Line <www.odontología online.com > [consulta: 28 Setiembre 2003]

22.Di Nasso, Patricia. Cuidado dental del niño con discapacidad. Boleten de la Asociación Argentina de odontología para niños, 2001; 30(4):21-24. 